\title{
Performance Evaluation of IPv4/IPv6 Transition Mechanisms for Real-Time Applications using OPNET Modeler
}

\author{
Khalid EL KHADIRI ${ }^{1}$, Ouidad LABOUIDYA ${ }^{2}$, Najib ELKAMOUN ${ }^{3}$, Rachid HILAL ${ }^{4}$ \\ STIC Laboratory \\ Chouaib Doukkali University \\ El Jadida, Morocco
}

\begin{abstract}
The problem of the potential depletion of IPv4 addresses has given rise to the development of a new version of the Internet Protocol named IPv6. This version of the protocol offers many improvements, including an increase in the address space from $2^{32}$ to $2^{128}$ and improvements in security, mobility, and quality of service. However, the transition from the current version to the new version (IPv4 to IPv6) is complicated and cannot be performed in a short time. The size and complexity of Internet make this migration task extremely difficult and timeconsuming. The Internet Engineering Task Force (IETF) took into account this migration problem and proposed transition mechanisms as temporary solutions allowing IPv4 to coexist and operate in parallel with IPv6 networks. The dual stack, manual tunnel, and 6to4 automatic tunnel appear to be promising solutions depending on their characteristics and benefits. In this paper, we study the performance of these transition mechanisms on real-time applications (VoIP and Video Conferencing) using the network simulator OPNET Modeler. Performance parameters such as delay, delay variation, jitter, MOS, and packet loss are measured for these transition mechanisms. The obtained results showed that the dual stack transition mechanism gave better network performance than the tunneling mechanisms.
\end{abstract}

Keywords - Dual stack; manual tunnel; 6to4; OPNET; VoIP; video conferencing

\section{INTRODUCTION}

IPv4, the current version of the Internet Protocol (IP), is the version on which the Internet cloud is currently based [1]. IPv6 is the new version of the protocol, developed by the IETF, which has many advantages, including addressing, autoconfiguration, mobility, quality of service, and security [2]. Furthermore, IPv4 and IPv6 are two protocols that are not compatible. Therefore the transition from IPv4 to IPv6 is complicated and is not a project that will succeed overnight. The deployment of IPv6 can only be done gradually (step by step) and can be divided into three phases as follows. Phase I: IPv6 is an island in the IPv4 ocean, where IPv4 dominates the global network. Phase II: IPv4 will become an island while IPv6 will be oceanic a few years later. That means that at this point IPv6 will be much wider-ranging than IPv4. The final phase, Phase III: most networks will be in native IPv6. During the transition phase from IPv4 to IPv6, a number of transition and migration mechanisms, proposed and implemented by the
IETF, can be used. Most of them have advantages and disadvantages depending on their deployments.

In this research paper, three IPv4/IPv6 transition mechanisms were examined. Namely: the dual stack, the manual tunnel, and the 6to4 automatic tunnel. These mechanisms were evaluated on a simulation network infrastructure under OPNET Modeler using two real-time applications (VoIP and Video Conferencing). The obtained results were compared with those of native IPv4 and IPv6 networks. A comparative analysis of the simulation results involves various parameters such as delay, delay variation, jigger, MOS, and packet loss. The remainder of the document is organized as follows. Section II will present a background of some IPv4/IPv6 transition mechanisms and their classification. Section III will discuss a non-exhaustive state of the art of the research work carried out in this field of research. The simulation scenarios for the chosen IPv4/IPv6 transition mechanisms will be described in Section IV. The results of the simulation and the comparative analysis will be discussed in Section V. The conclusions and perspectives will be presented in the final section of this paper.

\section{BACKGROUND}

During the transition phase to IPv6 and because of the incompatibility between IPv4 and IPv6 (the number of fields, the format, and the size of the addresses are different), several transition mechanisms to IPv6 have been developed. Each mechanism has specificities that make it suitable for a particular use. These mechanisms can be classified into three main families as shown in Fig. 1 below:

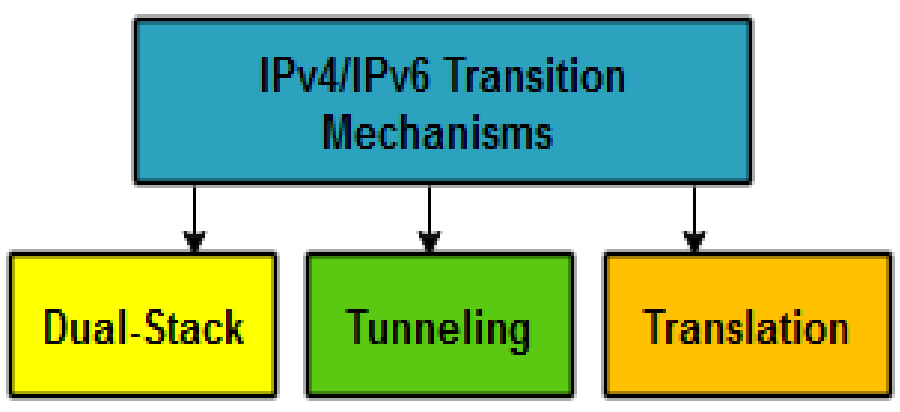

Fig. 1. IPv4/IPv6 transition mechanisms classification. 


\section{A. Dual Stack}

The dual stack [3] is the simplest transition mechanism to deploy. It requires that all network peripherals (computers, routers, servers, etc.) support both IPv4 and IPv6 protocols working in parallel and side by side. The applications communicate with IPv4 and IPv6. That means that we are on an IPv4/IPv6 network, and therefore we do not need additional mechanisms to access both IPv4 machines and IPv6 machines. In this case, the communications are transmitted by the IP layers corresponding to used addresses, and there is no conversion problem. The choice of the IP version is based on the result of the Domain Name System (DNS) query or application preference.

\section{B. Tunneling}

As IPv4 and IPv6 headers are different from each other, the IPv6 packet must be tunneled to head for its destination point across the incompatible IPv4 network. Tunneling [4] is a technique in which one protocol is encapsulated in another protocol depending on the network where the packet must be routed. In the case of an IPv6 tunnel, if an IPv6 source communicating with an IPv6 destination and an IPv4 network is between them, the IPv6 packets must be encapsulated in IPv4 headers in order to be routed for over the IPv4 network and reach their IPv6 destination.

\section{1) Manual Tunnel}

Here, IPv6 data are encapsulated in IPv4 packets and then transferred through the tunnel. At the endpoint of the tunnel, the packets will be decapsulated and transmitted to their destination [5]. The endpoint address of the tunnel is determined from the configuration information stored on the encapsulation/decapsulation points of the tunnel. These tunnels can be used from router to router, from host to router, from host to host or from router to host.

\section{2) 6to4 Automatic Tunnel}

This one allows communication between two IPv6 sites over an IPv4 network without needing for an explicitly configured tunnel or an IPv6-IPv4 compatible address. Thus, the tunnel end-node configuration is minimal [6]. Other tunneling mechanisms can be used such as: an IPv4-compatible automatic IPv6 tunnel [7], 6over4 [8], ISATAP [9], 6rd [9], etc.

\section{Translation}

This mechanism works by allowing a native IPv6 network to communicate with nodes on an IPv4 network and vice versa. That is an intermediate peripheral or service that converts packets headers to the border network.

\section{1) Network Address Translation-Protocol Translation} (NAT-PT)

It is a device residing at the edge of an IPv4/IPv6 network allowing communication between IPv4 nodes residing in an IPv4 network and IPv6 nodes residing in an IPv6 network and vice versa by translating IP addresses [10]. NAT-PT maintains a globally routable range of IPv4 addresses and assigns IPv4 addresses to IPv6 nodes and vice versa.
2) Dual Stack Application Level Gateway (DS-ALG)

Here, a dual stack IP peripheral is used and it can access IPv4 and IPv6 services in native mode [11]. It is a simple and robust solution, but it requires that all customers are configured to use ALG, and it only works for specific applications supported by ALG. Other translation mechanisms can be used such as: BIH [12], NAT64 [13]/DNS64 [14], SIIT [15], etc.

\section{RELATED WORKS}

Performance evaluation of IPv4/IPv6 transition mechanisms is a very active field of research. Several research works have been carried out in this area. Here, we present some of the most relevant ones.

The author Quintero and his colleagues compared the performance of three transition mechanisms, namely ISATAP, 6to4, and NAT64 with IPv4 and IPv6 networks [16]. This comparison in terms of delay and throughput was performed on different operating systems (Debian, Windows 7, Windows 8 and Windows 10) for two types of traffic: TCP and UDP. The obtained results showed that native IPv4 gave better performance, closely followed by native IPv6. The difference is mainly due to the length of the IP header ( 20 bytes in IPv4 compared with 40 bytes in IPv6). The selected tunneling solutions (ISATAP and 6to4) have similar performance in terms of delay and throughput. They represent the low level of the technologies studied on account of the additional IPv4 header added by the tunnel.

In [17], the author $\mathrm{Lu}$ and his colleagues carried out a performance evaluation in terms of a routing path between two mechanisms that are the 4over6 and dual stack. Consequently, the authors found that the experimental routing performance of a dual stack mechanism gave better performance than 4over6. The 7-node routing path with dual stack was better than 4over6 by $17,329 \%$.

In a detailed manner, the authors El Khadiri and his colleagues [18] and the author Govil [19] realized comparative studies of the mechanisms of the transition from IPv4 to IPv6 in order to help, among others, the customers, who want to use or connect using IPv6, to choose the suitable mechanism according to their needs. The study showed that the right solution for a particular customer or organization depended on its existing infrastructure and the time it takes to migrate to IPv6.

Other performance comparisons between IPv4, IPv6 networks, and the 6to4 tunnel have been discussed in [20], [21]. VoIP has been used as test traffic according to certain parameters such as delay, queuing delay, response time, jitter and throughput. Consequently, IPv4 gave better performance than IPv6. The difference is due to the IPv6 header length, which is higher than the one of IPv4. Similarly, these networks (IPv4 and IPv6 networks) gave better results compared with the 6to4 tunnel. This difference is due to the additional header added by the tunnel during the encapsulation/decapsulation operations of IPv6 packets in IPv4. However, these works did not take into account the impact of packet loss, which is considered as a very important parameter in the measure of performance of a network, on the one hand between IPv4 and IPv6 networks, and on the other hand related to the tunnel. 
In view of this reflexive synthesis, it turned out to be indispensable to complete the previous works. On one hand, by adding other IPv4/IPv6 transition mechanisms and on the other hand, we intend to evaluate the behavior of real-time applications, such as VoIP and Video Conferencing, taking into account the impact of the packet loss rate on the performance of the mechanisms that we are going to evaluate.

\section{SCENARIOS OF THE SIMULATION}

\section{A. Simulation Environment}

The proposed simulation network topology has been configured for five different scenarios. Three of them concern the dual stack transition mechanisms, manual tunnel, and 6to4 automatic tunnel. The remaining two scenarios target native IPv4 and IPv6 networks if all communications are respectively only IPv4 or IPv6. Our simulation was implemented using the simulation tool OPNET (Optimized Network Engineering Tool) as shown by the simulation network topology represented in Fig. 2 below. Two IPv6 sites (the first is set in Rabat and the other in Tangier) want to communicate with each other through an IPv4 backbone.

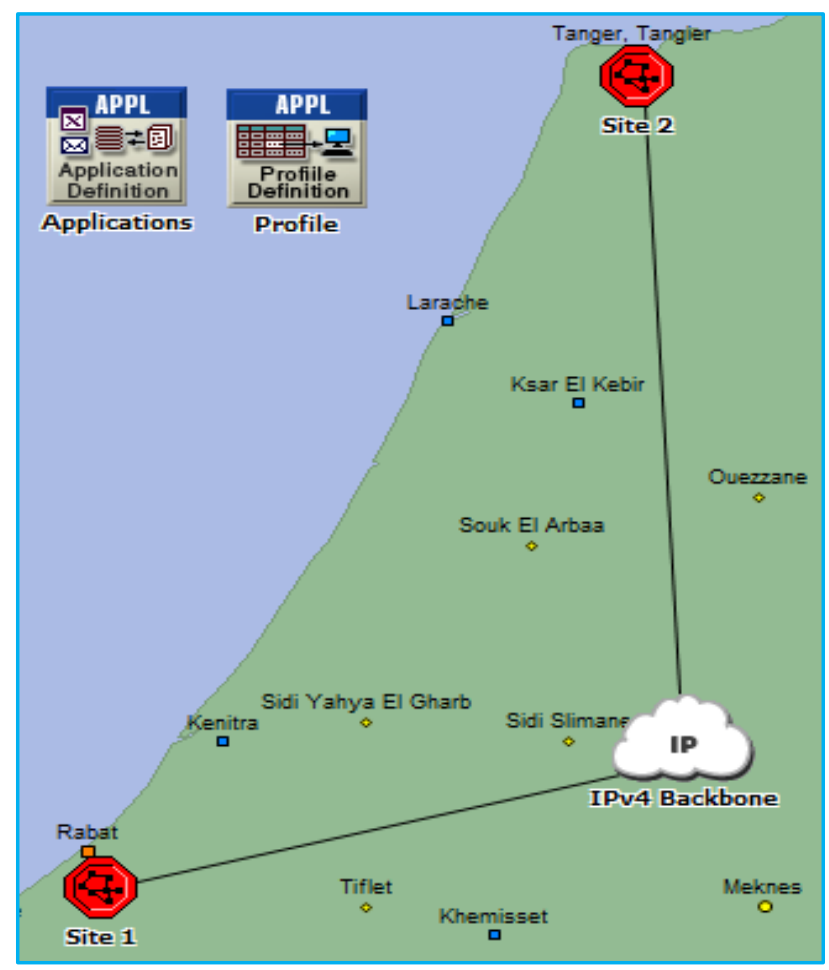

Fig. 2. The typology of simulation network.

To that end, we used the version 17.5 of OPNET (Riverbed Modeler Academic Edition) [22]. In order to simulate the different chosen IPv4/IPv6 transition techniques, we used realtime applications (VoIP and Video Conferencing) as test traffic. Regarding the routing, we have configured RIPv2 routing on the IPv4 backbone and RIPng routing on the IPv6 sites.

\section{B. Simulation Parameters}

Five simulation parameters were used during this simulation as follows:
1) End-to-End Delay: This parameter represents the endto-end delay that is measured between the time a packet is created and sent from a source until it is received at its destination.

2) Delay Variation: This is the variation between the unidirectional delay of selected packets in the same packet stream [23]. It is based on the end-to-end delay difference of these selected packets. This measure has a significant impact assessing the quality of voice/video conferencing applications.

3) Jitter: Jitter is defined as the end-to-end transmission delay difference between selected packets in the same packets stream, without taking into account of eventually lost packets [23], [24]. This parameter is important for a voice application because if the transmission delay varies for a VoIP conversation, the voice quality will be degraded. The best jitter value is the one closest to zero.

4) MOS: That is an important indicator for assessing the quality of a voice application [24] by giving it one of these values $(1,2,3,4,5)$ where 5 indicates excellent quality and 1 indicates poor quality.

5) Loss Rate: That is the number of lost packets in percent compared with sent packets.

\section{SIMULATION RESULTS AND ANALYSIS}

In this section, we present the results of the simulation by analyzing and comparing the five scenarios. For each specific case, the simulation process lasted 300 seconds using DES (Discrete Event Simulation). OPNET calculates the desired value at all times for the simulation time. In this simulation, the average values were monitored from the OPNET results viewer and exported to Excel in order to draw bar graphs for comparison purposes.

\section{A. End-to-End Delay}

The obtained results in Fig. 3 below represent the Video Conferencing Packet End-to-End Delay in milliseconds for the five proposed scenarios.

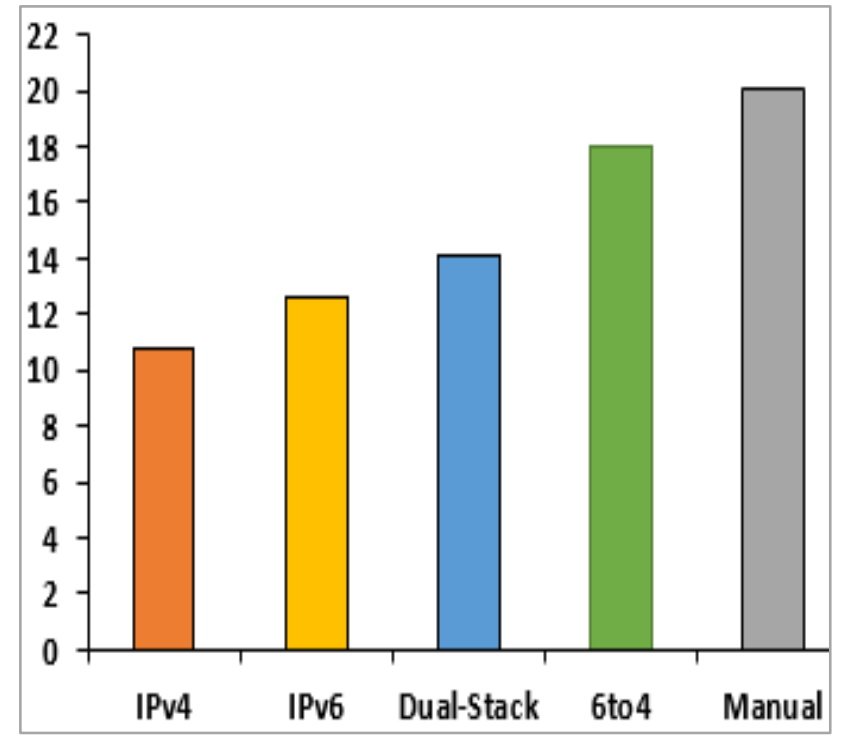

Fig. 3. Video conferencing packet end-to-end delay (ms). 
These results show that the dual stack is better than the other two IPv4/IPv6 transition mechanisms: with an average delay value of about $14 \mathrm{~ms}$ for the dual stack compared with $18 \mathrm{~ms}$ and $20 \mathrm{~ms}$ for the 6 to 4 tunnel and manual tunnel. That is due to the delay caused by encapsulation and decapsulation processes in the tunneling mechanisms whereas, in the dual stack, the two protocols simultaneously work without involving encapsulation or decapsulation. The comparison between the two protocols in relation to the same criterion indicates that IPv4 has better performance than IPv6. Indeed, IPv4 presents an average delay value of about $10.8 \mathrm{~ms}$ compared with $12.6 \mathrm{~ms}$ for IPv6. That is due to the IPv6 header length, which is higher than the one of IPv4.

Fig. 4 below shows the results of the Voice Packet End-toEnd Delay in milliseconds for the five scenarios.

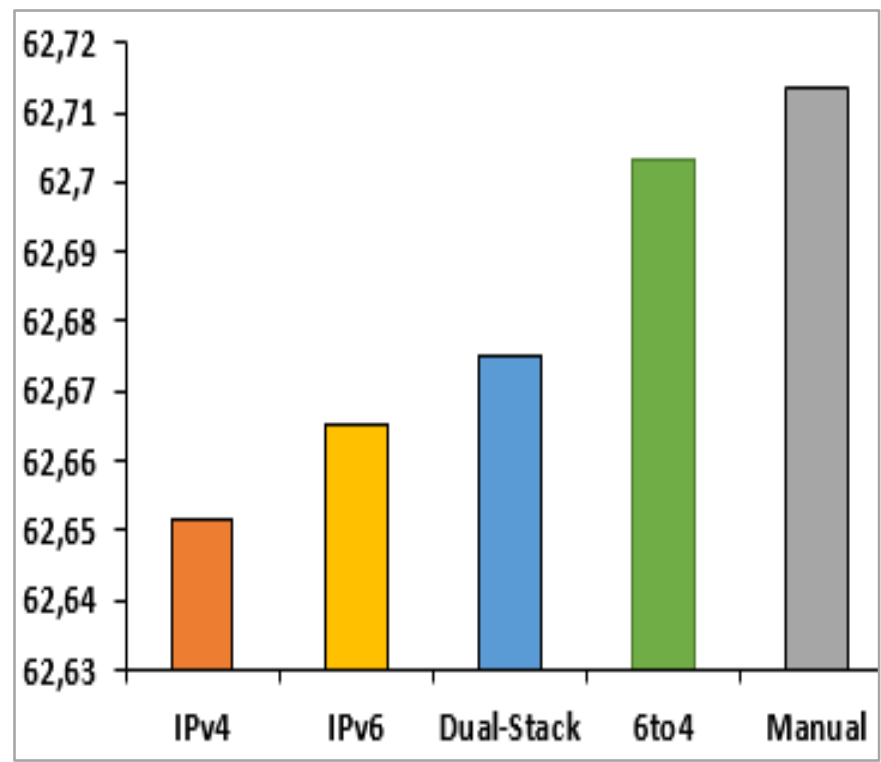

Fig. 4. Voice packet end-to-end delay (ms).

These results indicate that tunneling mechanisms recommend average delay values higher than those of dual stack. The difference is due to the additional IPv4 header that is added by tunneling for encapsulation/decapsulation operations. The comparison between the two protocols shows that IPv6 provides an average delay value higher than the one of IPv4. That is due to the IPv6 header length that is higher than the one of IPv4.

\section{B. Delay Variation}

Fig. 5 and 6 below represent the results of the Packet Delay Variation in milliseconds for the two real-time applications (Video conferencing and VoIP) for the five scenarios.

According to a first reading, it is clear that the dual stack is more performing than manual tunneling mechanisms and 6to4. Indeed, it presents a lower value in terms of Packet Delay Variation compared with the tunneling mechanisms. The comparison between the two protocols showed that IPv4 provides a lower delay variation than IPv6. That indicates that IPv4 provides a better quality regarding VoIP and Video Conferencing applications compared with IPv6.

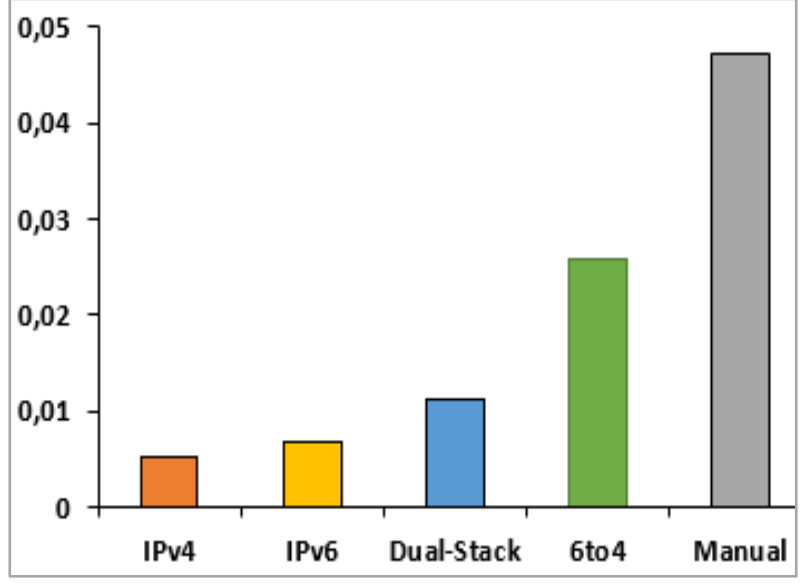

Fig. 5. Video conferencing packet delay variation (ms).

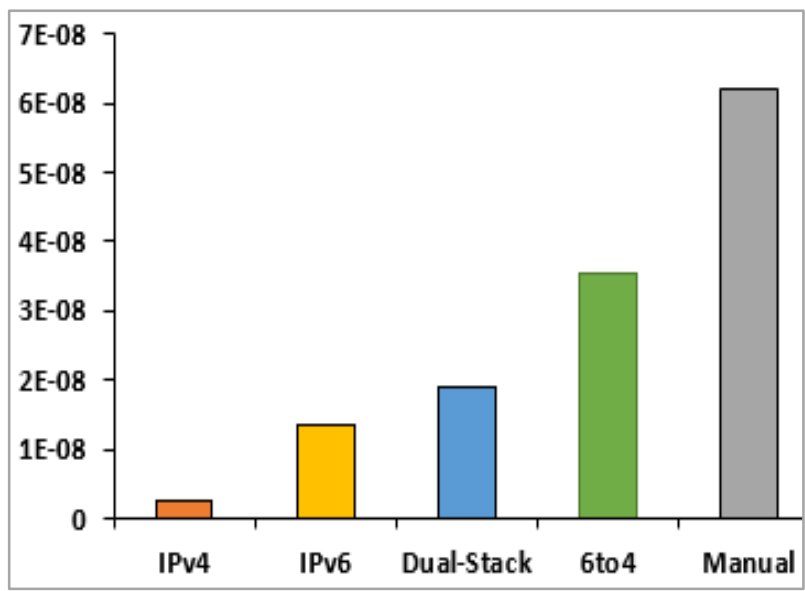

Fig. 6. Voice packet delay variation (ms).

\section{Jitter}

Fig. 7 below shows the average values of the Voice Jitter in milliseconds for the five scenarios.

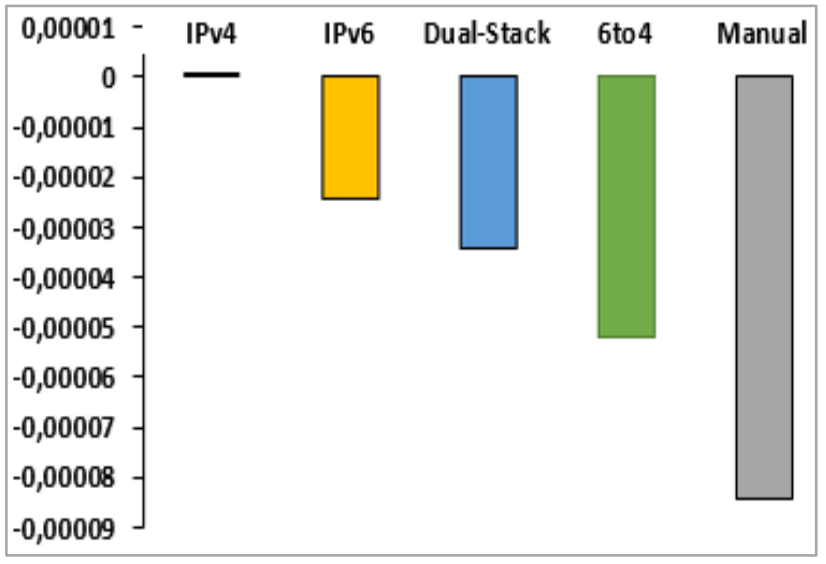

Fig. 7. Voice Jitter (ms).

These results indicate that the dual stack is better than the other two IPv4/IPv6 transition mechanisms. Indeed, it presents a lower average jitter value than those of the manual tunneling mechanisms and 6to4. The comparison between the two protocols in relation to the same criterion indicates that IPv4 is 
better than IPv6. That indicates that IPv4 offers better quality regarding VoIP applications compared with IPv6.

\section{D. $M O S$}

The obtained results in Fig. 8 below represent the average MOS values for the five scenarios. The highest MOS value indicates one better performance. As it can be seen, the results indicate that dual stack provides better voice quality than the other two IPv4/IPv6 transition mechanisms that appeared similar. The comparison between the two protocols in relation to the same criterion indicates that IPv4 has better voice quality than IPv6.

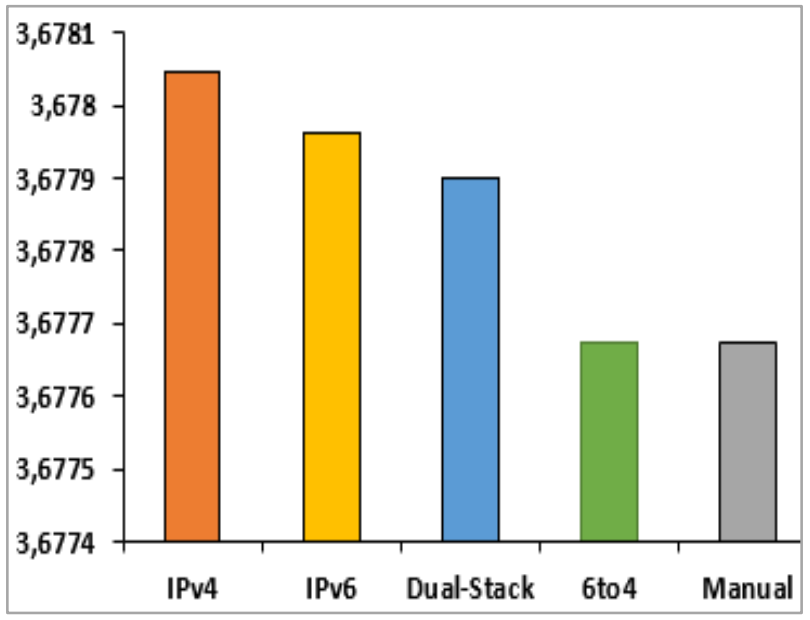

Fig. 8. Voice MOS.

\section{E. Loss Rate}

Fig. 9 below shows the results of the Video Conferencing Packet Loss Rate for the five scenarios. As it can be seen, the results show that the packet loss rate of manual and 6to4 tunneling mechanisms is higher than the one of the dual stack: a loss rate of about $3,6 \%$ and $4,1 \%$ for respectively 6 to 4 and manual tunneling mechanisms compared with $2,7 \%$ for the dual stack. That is due to the encapsulation/decapsulation operations of IPv6 packets encapsulated in IPv4 packets by the tunneling mechanisms. IPv4 presents a lower loss rate than IPv6.

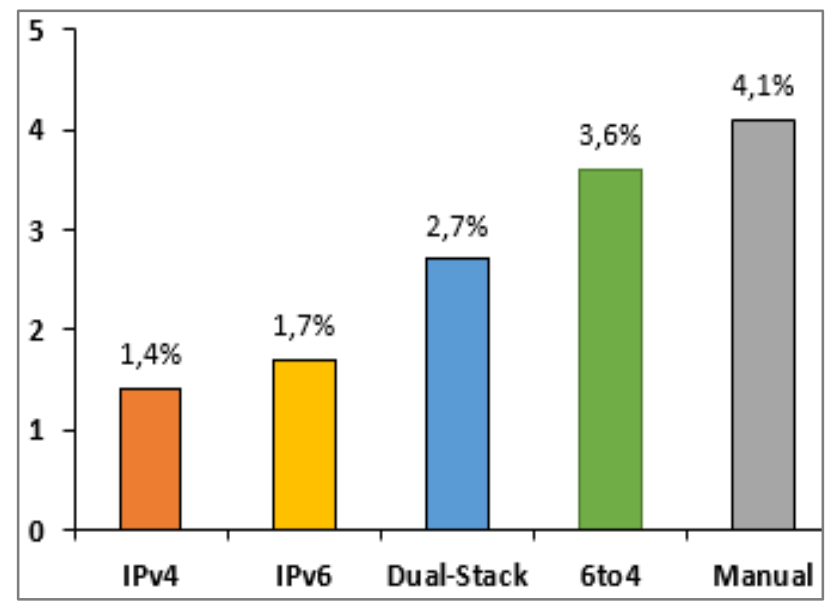

Fig. 9. Video conferencing packets loss rate (\%).
Fig. 10 below shows the results of the Voice Packets Loss Rate for the five scenarios. As it can be seen, the results reveal that the loss rate of the dual stack is lower than the one of the tunneling mechanisms: a loss rate of about $2,3 \%$ for the dual stack against respectively $3,2 \%$ and $3,7 \%$ for the 6 to 4 and manual tunneling mechanisms. The difference is due to encapsulation/decapsulation operations of IPv6 packets encapsulated in IPv4 packets by tunneling mechanisms. The comparison between the two protocols indicates that IPv4 presents a lower loss rate than IPv6.

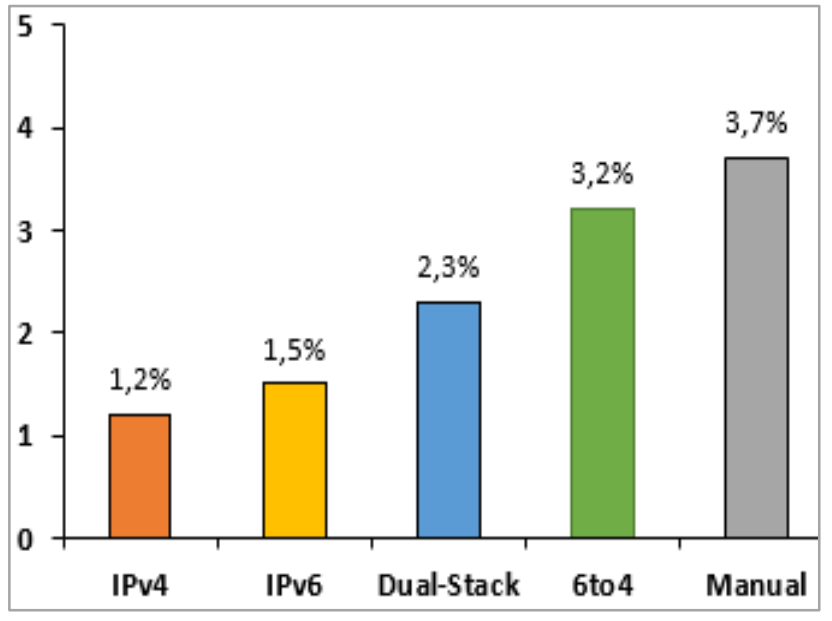

Fig. 10. Voice packets loss rate (\%).

\section{CONCLUSIONS AND PERSPECTIVES}

In this article, we studied and evaluated three IPv4/IPv6 transition mechanisms: the dual stack, the manual tunnel, and the 6to4 automatic tunnel. These mechanisms were evaluated on a simulation network infrastructure under OPNET Modeler using two real-time applications (VoIP and Video Conferencing). The obtained results were compared with those of native IPv4 and IPv6 networks in terms of delay, delay variation, jitter, MOS, and packets loss.

According to our results, the dual stack mechanism presents better performance compared with the two studied tunneling mechanisms: the manual tunnel and the 6to4 automatic tunnel. The performance degradation of these lasts is due to the additional IPv4 header added by tunneling during encapsulation/decapsulation operations of IPv6 packets in IPv4 packets. In contrast, in the dual stack, the two IP stacks work in parallel and side by side without involving encapsulation or decapsulation.

In terms of deployment, the dual stack mechanism requires that all network peripherals take over both protocols (IPv4 and IPv6), which is difficult in the case of a large network that will require many configurations, including problem-solving in case of a crash. Consequently, this transition mechanism can be deployed in a small network. In contrast, tunneling mechanisms represent a good choice for large networks where only both ends of the network have to be configured with IPv4 and IPv6 protocols.

Regarding both protocols (IPv4 and IPv6), the results showed that IPv4 presents better performance than IPv6. Indeed, that is the price to pay for resolving the problem of the 
potential depletion of the IPv4 addresses. The difference is actually due to the IPv6 header length, which is higher than the one of IPv4 (40 bytes in IPv6 compared with 20 bytes in IPv4).

Our study was limited by the OPNET Modeler simulator, which only supports the study of integrated IPv4/IPv6 transition mechanisms (the ones we studied). Our future research work focuses on an experimental evaluation of IPv4/IPv6 transition mechanisms by adding other mechanisms and by studying their performance for various types of applications.

\section{REFERENCES}

[1] J. Postel, "Internet protocol," RFC 791, Sep. 1981.

[2] R. Hinden, "Internet protocol, version 6 (IPv6) specification," RFC 8200, 2017.

[3] S. Aravind and G. Padmavathi, "Migration to Ipv6 from IPV4 by dual stack and tunneling techniques," in Smart Technologies and Management for Computing, Communication, Controls, Energy and Materials (ICSTM), 2015 International Conference on, 2015, pp. 107-111.

[4] J. L. Shah and J. Parvez, "An examination of next generation IP migration techniques: Constraints and evaluation," in Control, Instrumentation, Communication and Computational Technologies (ICCICCT), 2014 International Conference on, 2014, pp. 776-781.

[5] S. Steffann, I. van Beijnum, and R. van Rein, "A Comparison of IPv6over-IPv4 tunnel mechanisms,” RFC 7059, 2013.

[6] A. Misra and H. Chawla, "Performance Analysis of IPv6 Dual-Protocol Stack and Tunnel Transition," Int. J. Sci. Eng. Technol. Res. IJSETR Vol., vol. 5, 2016.

[7] B. Chhetri, "Transition from IPV4 to IPV6," 2015.

[8] R. Kaur, S. Kumar, and V. K. Patle, "Analysis of Tunneling Transition Mechanism in IPv6," Res. J. Sci. Technol., vol. 5, no. 3, p. 3, 2013.

[9] G. LENCSE and Y. KADOBAYASHI, "Survey of IPv6 transition technologies for security analysis," in IEICE Technical Committee on Internet Architecture (IA) Workshop, Tokyo Japan, 2017, pp. 19-24.

[10] A. H. A. Mohamed, A. B. Abdelnabi, and H. E. Edris, "Performance Analysis of IPv4, IPv6 Transition Technologies," 2016.

[11] A. Singh, A. Maheshawari, and D. Shukla, "Study for IPv4 and IPv6 Coexistence," 2013.
[12] B. Huang, H. Deng, and T. Savolainen, "Dual-stack hosts using" bumpin-the-host"(BIH),, 2012.

[13] M. Bagnulo, P. Matthews, and I. van Beijnum, "Stateful NAT64: Network address and protocol translation from IPv6 clients to IPv4 servers," RFC 6146, 2011.

[14] M. Bagnulo, P. Matthews, A. Sullivan, and I. van Beijnum, "DNS64: DNS extensions for network address translation from IPv6 clients to IPv4 servers," RFC 6147, 2011.

[15] J. Hanumanthappa and H. Annaiah, "DW\&C: Dollops Wise Curtail IPv4/IPv6 Transition Mechanism using NS2," ArXiv Prepr. ArXiv14093767, 2014.

[16] A. Quintero, F. Sans, and E. Gamess, "Performance evaluation of IPv4/IPv6 transition mechanisms," Int. J. Comput. Netw. Inf. Secur., vol. 8, no. 2, p. 1, 2016.

[17] T. Te Lu, C. Y. Wu, W. Y. Lin, H. P. Chen, and K. P. Hsueh, "Comparison of IPv4-over-IPv6 (4over6) and Dual Stack Technologies in Dynamic Configuration for IPv4/IPv6 Address," in Advances in Intelligent Information Hiding and Multimedia Signal Processing, Springer, 2017, pp. 259-269.

[18] K. EL KHADIRI, O. LABOUIDYA, N. ELKAMOUN, and R. HILAL, "Etude comparative des mécanismes de transition de l'IPv4 à l'IPv6," Mediterranean Telecommunications Journal, vol. 7, no. 1, Jan. 2017.

[19] J. Govil, J. Govil, N. Kaur, and H. Kaur, "An examination of IPv4 and IPv6 networks: Constraints and various transition mechanisms," in Southeastcon, 2008. IEEE, 2008, pp. 178-185.

[20] A. Ahmed, A. Mustafa, and G. Ibrahim, "Performance Evaluation of IPv4 Vs Ipv6 and Tunnelling Techniques Using Optimized Network Engineering Tools (OPNET)," IOSR Journal of Computer Engineering (IOSR-JCE), vol. 17, no. 1, 2015.

[21] A. Salam and M. A. Khan, "Performance Analysis of VoIP over IPV4, IPv6 and 6-to-4 Tunneling Networks," (IJCSIS) International Journal of Computer Science and Information Security, vol. 14, no. 6, 2016.

[22] "Modeler OP. Riverbed Technology. Inc. https://www.riverbed.com," 2018.

[23] K. Neupane, V. Kulgachev, A. Elam, S. H. Vasireddy, and H. Jasani, "Measuring the performance of VoIP over Wireless LAN," in Proceedings of the 2011 conference on Information technology education, 2011, pp. 269-274.

[24] O. Slavata, J. Holub, and P. Hübner, "Impact of jitter and jitter buffer on the final quality of the transferred voice," in Wireless Systems (IDAACSSWS), 2012 IEEE 1st International Symposium on, 2012, pp. 120-123. 